\title{
Bouncing cosmologies via modified gravity in the ADM formalism: Application to Loop Quantum Cosmology
}

\author{
Jaume de Haro*1,2 and Jaume Amorós ${ }^{\dagger 2}$ \\ ${ }^{1}$ Departament de Matemàtiques, Universitat Politècnica de Catalunya, Colom 11, 08222 Terrassa, Spain \\ ${ }^{2}$ Departament de Matemàtiques, Universitat Politècnica de Catalunya, Diagonal 647, 08028 Barcelona, Spain
}

\begin{abstract}
We consider the ADM formalism as a tool to build bouncing cosmologies. In this approach, the foliation of the spacetime has to be fixed in order to go beyond General Relativity modifying the gravitational sector. Once a preferred slicing, which we choose based on the matter content of the universe following the spirit of Weyl's postulate, has been fixed, $f$ theories depending on the extrinsic and intrinsic curvature of the slicing are covariant for all the reference frames preserving the foliation, i.e., the constraint and dynamical equations have the same form for all these observers. Moreover, choosing multivalued $f$ functions, bouncing backgrounds emerge in a natural way. In fact, the simplest is the one corresponding to holonomy corrected Loop Quantum Cosmology. The final goal of this work is to provide the equations of perturbations which, unlike the full equations, become gauge invariant in this theory, and apply them to the so-called matter bounce scenario.
\end{abstract}

PACS numbers: 04.20.Fy, 04.50.Kd, 98.80.Jk.

Keywords: Modified gravity, ADM formalism, Loop Quantum Cosmology, Weyl postulate.

\section{INTRODUCTION}

Bouncing cosmologies (see the reviews [1 $[$ ) seem to be the most natural alternative to the inflationary paradigm [6] 8], although in some papers [9-12] it seems to be only an implementation of it, introducing a pre-inflationary dynamics in order to remove the initial singularity. However, it is not an easy task to build up this kind of scenarios in the framework of the General Relativity (GR) 13 17, or to go beyond GR using modified theories such as $f(R)$ gravity (see for example [18 20]), modified Gauss-Bonnet gravity 21 23, or Born-Infeld inspired gravity theories [24, 25. A simple non-singular bounce, which replaces the Big Bang singularity, emerges from holonomy corrected Loop Quantum Cosmology (LQC) [26 35].

The LQC theory, in the flat Friedmann-Lemaitre-Robertson-Walker (FLRW) spacetime, containing only holonomy corrections (not inverse-volume effects) can be built as follows: since the gravitational part of the classical Hamiltonian contains the Ashtekar connection, namely $\mathfrak{c}=\beta \dot{a}$, where $\beta$ is the Immirzi parameter [36] and $a$ the scale factor, which does not have a well defined quantum operator (see for instance [37, 38]), so one has to express it in terms of holonomies in order to obtain a quantity able to be quantized which captures the underlying loop quantum dynamics (see for instance [39]). At effective level, as has been shown in [40, 41], this is equivalent to replacing the Ashtekar connection by the function $\sin (\mu \mathrm{c}) / \mu$, where $\mu$ is a parameter with dimensions of length. Then, with the new holonomy corrected Hamiltonian constraint one obtains the modified LQC-Friedmann equation, which in the phase space $(H, \rho)$ represents an ellipse [42, where $H$ is the Hubble parameter and $\rho$ the energy density, and thus, contrarily to GR where this curve is an unbounded parabola, singularities such as the big bang, big rip [43] or little rip [44] are directly removed.

Given the success of the effective theory for homogeneous and isotropic space-times, it is natural to try the same approach for cosmological perturbations in LQC. If one simply replaces the Ashtekar connection everywhere it appears in the Hamiltonian constraint by a sinusoidal function $\mathfrak{c} \rightarrow \frac{\sin (n \mu \mathfrak{c})}{n \mu}$ where $n$ is a natural number [45, the result is a theory with anomalies in its constraint algebra. A more careful treatment that gives an anomaly-free theory in the longitudinal gauge has been found [46], and this has since been extended to the gauge-invariant case by allowing for new terms in the holonomy modified Hamiltonian that vanish in the classical limit [47-50]). The result is a modified Mukhanov-Sasaki equation that can be used to study the dynamics of cosmological perturbations in LQC.

\footnotetext{
* E-mail: jaime.haro@upc.edu

$\dagger$ E-mail: jaume.amoros@upc.edu
} 
On the other hand, it is well-known that the background of holonomy corrected LQC in flat FLRW spacetime can be easily mimicked in modified gravity if one has an scalar field that only depends on the square of the Hubble parameter 51533. This scalar appears in teleparallelism, where the spacetime is equipped by the Weitzenböck connection [54] and an orthonormal basis parallelaly transported is chosen in the tangent bundle (see for example [55, 56]). For a FLRW geometry, using synchronous co-moving coordinates, the scalar named torsion and denoted by $\mathcal{T}$, is given by $\mathcal{T}=-6 H^{2}$ [57, 58. Then, the $f(\mathcal{T})$ theory that leads to the same ellipse as holonomy corrected LQC could be named teleparallell LQC [61]. Dealing with perturbations, the perturbed equations in teleparallelism [59, 60] have been applied to this particular function in 62, 63, obtaining a different kind of equation, which differs from the ones of holonomy corrected LQC starting with the velocity of the speed of sound. Unfortunately, besides avoiding the usual Levi-Civita connection, the scalar torsion is not local Lorentz invariant, that is, a local Lorentz transformation of the orthonormal basis could lead to a new orthogonal basis, in which the value of the scalar torsion is different form the one obtained using the first basis [64, 65. Thus, given a torsion, a $f(\mathcal{T})$ theory becomes preferred frame, which is an undesirable feature. Effectively, let $\left\{\mathbf{e}_{\nu}\right\}_{\nu=0, . .3}$ and $\left\{\overline{\mathbf{e}}_{\nu}\right\}_{\nu=0, . .3}$ be two orthonormal basis in the tangent bundle parallelally transported, and let $\mathcal{T}$ and $\overline{\mathcal{T}}$ be their corresponding torsions. Since its difference is a divergence, when $f$ is the identity their corresponding actions are the same, which will not happen for a general function $f$.

In the same way as in teleleparallelism, in the Arnowitt-Deser-Misner (ADM) formalism of GR [66, 67, where a globally hyperbolic spacetime could be foliated by three-dimensional space-like sub-manifolds parameterized by a time and the connection is the usual Levi-Civita one, the extrinsic curvature scalar, namely $\mathcal{I}$, is also equal to $-6 H^{2}$ in the flat FLRW spacetime using synchronous co-moving coordinates. Since the scalar curvature of the full manifold, namely $R$, is equal to $\mathcal{R}+\mathcal{I}$ plus a divergence, where $\mathcal{R}$ is the intrinsic curvature, i.e., the scalar curvature of the sub-manifolds, on can built some classes of $F(\mathcal{R}, \mathcal{I})$ gravitational theories, leading to different backgrounds and one of them will be the same as in holonomy corrected LQC. In this way, the main goal of this work is to provide the gravitational equations in a particular case, namely $F(\mathcal{R}, \mathcal{I})=\mathcal{R}+f(\mathcal{I})$, its perturbed equations and, as a particular case, to calculate explicitly them for the $f$ theory that leads to the same background as LQC. At this point, it is important to emphasize that the theory is gauge dependent, in the sense that, it depends on the foliation chosen or, equivalently, on the choice of the normal vector, because only the sum of the intrinsic and extrinsic curvature is the same, up a divergence, for all the slicing. So, when the gauge is fixed, the theory becomes covariant only for the coordinate systems preserving the foliation, i.e., the equations only will become with the same aspect in these reference frames. Since cosmology deals with the evolution of the universe, in order to have a well defined Cauchy problem [67, 68] one has to fix precisely a foliation, and of course there are some gauges such as the so-called "geodesic slicing" 69, where the lapse function is chosen to be 1, the constant main curvature (the trace of the extrinsic curvature tensor) slicing [70, the maximal slicing [71, which corresponds to the vanishing of the mean curvature of the hyper-surfaces $\Sigma_{t}$, or the harmonic slicing [72, where the $t$ coordinate is required to be harmonic with respect to the D'Alembertian. In this work, as in the Chapter 4 of [73, and following the spirit of Weyl's principle (see [74] for a historical and critical review), we argue that there is a preferred 4-velocity field in the whole spacetime, which generates a preferred non-crossing family of world-lines. Therefore, taking the parameter $t$ as the proper time of these preferred observers one obtains a preferred synchronous gauge, or choosing the time $t$ defining the so-called co-moving slicing, i.e., the slicing orthogonal to the world-lines 75 , which, as we will see, is the best choice when the universe is filled by an scalar field, one obtains two preferred different $3+1$ splittings of the spacetime. Fortunately, and it is a nice feature of the theory, the equations of perturbations are gauge invariant, i.e., slicing independent, provided that the background, which is the FLRW spacetime, has been split as in GR, using co-moving coordinates.

The manuscript is organized as follows: In section II we review the ADM formalism and, for a given foliation, we generalize GR to a $\mathcal{R}+f(\mathcal{I})$ gravitational theory, obtaining the particular $f$ that leads to the same background as holonomy corrected LQC. At the end of the Section, the gravitational equations that generalize those of GR are derived. Section III is devoted to the study of scalar perturbations in this theory, comparing them with those of the teleparallel $f(\mathcal{T})$ gravity, and also applying them to LQC. In section IV we do the same as in section III but for tensor perturbations, and finally, in section $\mathrm{V}$ we apply our previously obtained results to the matter bounce scenario in LQC.

The units used throughout the paper are $\hbar=c=1$, where $M_{p l}=\frac{1}{\sqrt{8 \pi G}}$ is the reduced Planck's mass.

\section{ADM FORMALISM REVISITED}

In order to deal with bouncing cosmologies without initial or final singularities we assume that the spacetime is globally hyperbolic, i.e., it admits a three-dimensional space-like Cauchy surface $\Sigma$, four-dimensional Lorentz manifold 
$\mathcal{M}$ with metric $g$ (see [76] or the Chapter 3 of [67] for a review of these concepts), and thus, following the Geroch's splitting theorem [77, topologically the spacetime is homeomorphic to $\Sigma \times \mathbb{R}$ and thus it admits a foliation or slicing $\left(\Sigma_{t}\right)_{t \in \mathbb{R}}$ where for $t \in \mathbb{R}$

$$
\Sigma_{t} \equiv\{p \in \mathcal{M}: \hat{t}(p)=t\}
$$

being $\hat{t}: \mathcal{M} \rightarrow \mathbb{R}$ a scalar field defined in the full spacetime.

As in the Arnowitt-Deser-Misner (ADM) formulation of GR [66, we make the decomposition $\partial_{t}=N \mathbf{n}+\mathbf{N}$ where $N$ is the so-called lapse function, $\mathbf{N}$ is a vector belonging in the tangent space of $\Sigma_{t}$ named the shift vector and $\mathbf{n}$ is a vector perpendicular to $\Sigma_{t}$, satisfying $g(\mathbf{n}, \mathbf{n})=-1$. Now, let $\left\{\partial_{j}\right\}_{j=1,2,3}$ be a basis of the tangent space of $\Sigma_{t}$, then the metric, in coordinates, is given by

$$
d s^{2}=g_{\mu \nu} d x^{\mu} d x^{\nu}=-N^{2} d t^{2}+\gamma_{i j}\left(d x^{i}+N^{i} d t\right)\left(d x^{j}+N^{j} d t\right),
$$

where $\gamma_{i j}=g\left(\partial_{i}, \partial_{j}\right)$ are the entries of the induced metric in $\Sigma_{t}$, which we denote by $\gamma$, and $N^{i}$ are the coordinates of the vector $\mathbf{N}$ in the basis $\left\{\partial_{j}\right\}_{j=1,2,3}$, i.e., $\mathbf{N}=N^{j} \partial_{j}$.

An important ingredient of this formulation is the extrinsic curvature tensor which, although defined with a negative sign in some books, in this work is given by

$$
K(\mathbf{u}, \mathbf{v})=g\left(\nabla_{\mathbf{u}} \mathbf{v}, \mathbf{n}\right)
$$

where we have denoted by $\nabla$ the Levi-Civita connection, and $\mathbf{u}, \mathbf{v}$ are vectors of the tangent space of $\Sigma_{t}$.

Let $\mathcal{R}$ be the intrinsic curvature, i.e., the scalar curvature of $\Sigma_{t}$, and $\mathcal{I}=K_{i j} K^{i j}-(\operatorname{Tr}(K))^{2}$, where $\operatorname{Tr}(K)=K_{i}^{i}$ is the trace of the extrinsic curvature tensor. Then, the scalar curvature of the whole spacetime $\mathcal{M}$ is related with these quantities via the equality 67 .

$$
R=\mathcal{R}+\mathcal{I}-2 \nabla_{i} v^{i}
$$

where $\nabla_{i} v^{i}$, is the divergence of the vector field $\mathbf{v}=\nabla_{\mathbf{n}} \mathbf{n}-\nabla_{i} n^{i} \mathbf{n}$.

Therefore, the gravitational part of the Hilbert-Einstein action could be written as

$$
S_{H E ; \text { grav }}=\frac{M_{p l}^{2}}{2} \int_{\mathcal{M}} \sqrt{-g} R=\frac{M_{p l}^{2}}{2} \int_{t_{1}}^{t_{2}}\left\{\int_{\Sigma_{t}} N \sqrt{\gamma}(\mathcal{R}+\mathcal{I}) d x^{3}\right\} d t
$$

which could be generalized, as a $F$ gravitational theory, taking the action

$$
S_{F ; \text { grav }}=\frac{M_{p l}^{2}}{2} \int_{t_{1}}^{t_{2}}\left\{\int_{\Sigma_{t}} N \sqrt{\gamma} F(\mathcal{R}, \mathcal{I}) d x^{3}\right\} d t .
$$

Here, it is important to stress that this generalization depends on the chosen foliation. Effectively, for two different slicing, namely $\Sigma_{t}$ and $\bar{\Sigma}_{\bar{t}}$, the difference between $\mathcal{R}+\mathcal{I}$ and $\overline{\mathcal{R}}+\overline{\mathcal{I}}$ is a divergence, so, in GR, the action is the same, but when one considers a $F(\mathcal{R}, \mathcal{I})$ theory the action is different from one to other slicing. For this reason, as we have explained in the Introduction, we have to fix a foliation $\Sigma_{t}$ (the gauge) as in the Cauchy problem, and thus, once the foliation has been chosen, namely $\Sigma_{t}$, the action is covariant for all the coordinate systems where the foliation $\bar{\Sigma}_{\bar{t}}$ coincides with the chosen one, what always happens for transformations of the form $\bar{t}=g(t)$, where $g$ is an arbitrary function independent of the spatial coordinates.

To make physical sense of the theory, the fixed slicing has to be preferred in some sense, as it could be the frame at rest with respect the Cosmic Microwave Background. To justify our choice, we first remember the Weyl's postulate, which asserts: "The world-lines of galaxies (on average) form a bundle of non-intersecting geodesics orthogonal to a series of space-like hyper-surfaces. This series of hyper-surfaces allows for a common cosmic time and the space-like hyper-surfaces are the surfaces of simultaneity with respect to this cosmic time" [78. So, the idea behind the Weyl's postulate is to build up a co-moving reference frame in which the constituents of the universe are at rest (on average) relative to the co-moving coordinates. However, the basis of Weyl postulate (e.g. non-intersecting (on average) worldlines of galaxies) seems questionable if there exists some period in cosmic history when the universe is not filled by dust, for example in the radiation period when the word "galaxies" has to be changed by relativistic gas particles, or earlier. 
To overcome the difficulties of Weyl's postulate and develop it, we follow the Ellis proposal [79] (see also [80]), based on an specific choice of preferred fundamental world-lines whose 4-velocity, which following the spirit of the Weyl's postulate has to be intrinsically determined by the constituents of the universe, is the time-like eigenvector of the stress tensor, i.e., satisfyes $T_{\mu}^{\nu} u^{\mu}=\lambda u^{\nu}$. For realistic matter, it was shown (see page $89-90$ of [76]) that such family exists and is unique due to the weak energy condition: $T(\mathbf{v}, \mathbf{v}) \geq 0$ for any time-like vector $\mathbf{v}$. What is important is that if this is a 4-velocity field defined on the whole manifold it implies that its world-lines never cross due to the unicity of the solutions of a differential equation, and thus one has a $3+1$ splitting of the spacetime. Finally, the time could be the corresponding proper time of these world-lines defining a preferred synchronous gauge or the co-moving slicing defined as the foliation $\Sigma_{t}$ perpendicular to the world-lines. In fact, as we will show, when the he universe is filled by an scalar field $\phi$, the later prescription is the better one because in this case the slices coincide with the hyper-surfaces $\phi=t=$ constant, and thus, the matter component of the universe is not perturbed along a slice.

Note also that, this generalization is completely different from the standard modified $F(R)$ gravity, where the modified Friedmann equation is a dynamical equation which depends on the second derivative of the Hubble parameter (see for instance the reviews [81, 82,), because in our theory, as we will immediately see, the modified Friedmann equation is a constraint between de Hubble parameter, the energy density and the scale factor, that does not contain higher order derivatives.

As an special case in cosmology, assuming the isotropy and homogeneity of the universe, the Friedman-LemaittreRobertson-Walker (FLRW) geometry has to be used, whose line element for co-moving observers is given by

$$
d s^{2}=-N(t) d t^{2}+a^{2}(t) d \Sigma_{t}^{2}
$$

Now we consider the stress tensor for a perfect fluid $T_{\mu}^{\nu}=(\rho+P) u_{\mu} u^{\nu}+P \delta_{\mu}^{\nu}$ where $P$ is the pressure and $\mathbf{u}$ is the 4 -velocity of the observers. Since $T_{\mu}^{\nu} u^{\mu}=-p u^{\nu}$, this shows that the 4 -velocity is the time-like eigenvector of the stress tensor which leads to the preferred foliation in the FLRW geometry. In the same way, for an scalar field the stress tensor is $T_{\mu}^{\nu}=\partial_{\mu} \phi \partial^{\nu} \phi-\left(\frac{1}{2} g(\nabla \phi, \nabla \phi)+V(\phi)\right) \delta_{\mu}^{\nu}$ where $\nabla \phi$ is the gradient of the scalar field. Provided that the gradient was time-like, the 4-velocity $\mathbf{u}=-\frac{\nabla \phi}{\sqrt{-g(\nabla \phi, \nabla \phi)}}$ is an eigenvector, which for the flat FLRW geometry, has the form $\mathbf{u}=\left(\frac{\dot{\phi}}{N|\dot{\phi}|}, 0,0,0\right)$ and thus leads to the same foliation as the co-moving observers. In fact, when $N=1, t$ is the proper time of the observers and also the slice $t=$ constant are perpendicular to the world-lines of the observers, that is, for the FLRW spacetime with $N=1$, the co-moving and synchronous slicing coincide.

Note that, if one takes another foliation $\bar{\Sigma}_{\bar{t}}$ with $\bar{t}=g(t, \mathbf{x})$ and $g$ a function depending of the spatial coordinates $\mathbf{x}$, quantities such as the energy density, the intrinsic or extrinsic curvatures which are constant in every slice, become non-homogeneous in the new slices $\bar{t}=$ constant, and thus, introduce fictitious perturbations when the new slice is a consequence of a simple coordinate perturbation (see the beginning of section 7 in [83]).

Dealing with this preferred slicing, the extrinsic and intrinsic curvatures are:

$$
\mathcal{I}=-\frac{6 H^{2}}{N^{2}}, \quad \mathcal{R}=\frac{6 k}{a^{2}},
$$

where $k=0,1,-1$ for a flat, closed and open slice $\Sigma_{t}$, respectively.

In that case the total action would be

$$
S_{F ; \text { tot }}=\int_{t_{1}}^{t_{2}} N V \mathcal{L}_{\text {grav }} d t+\int_{t_{1}}^{t_{2}} N V \mathcal{L}_{m} d t
$$

where the volume is $V=a^{3}$, the gravitational piece of the Lagrangian is $\mathcal{L}_{\text {grav }}=\frac{M_{p l}^{2}}{2} F(\mathcal{R}, \mathcal{I})$, and the matter part for a barotropic fluid is $\mathcal{L}_{m}=-\rho(t)$ [84, 85], and $\mathcal{L}_{m}=P=\frac{1}{2 N^{2}} \dot{\phi}^{2}-V(\phi)$ for a scalar field minimally coupled with gravity, where $P$ is the pressure. As a function of the volume and its derivative one has

$$
\mathcal{I}=-\frac{2 \dot{V}^{2}}{3 N^{2} V^{2}}, \quad \mathcal{R}=\frac{6 k}{V^{2 / 3}} .
$$

Since the action does not depend on the derivative of the lapse function, the Euler-Lagrange equation becomes

$$
\partial_{N}\left(N\left(\mathcal{L}_{\text {grav }}+\mathcal{L}_{m}\right)\right)=0,
$$


which leads to the well-known Hamiltonian constraint, or equivalently, for $N=1$ to the modified Friedmann equation

$$
-2 \mathcal{I} F_{\mathcal{I}}+F=\frac{2 \rho}{M_{p l}^{2}} \Longleftrightarrow 2 H^{2} F_{\mathcal{I}}+\frac{1}{6} F=\frac{\rho}{3 M_{p l}^{2}},
$$

where we have denote by $F_{\mathcal{I}}$ the partial derivative of $F$ with respect to the extrinsic curvature.

We see that this is a constraint that defines a surface in the space $(a, H, \rho)$, and, in particular, for the flat FLRW metric a curve in the plane $(H, \rho)$.

The dynamical equation is obtained from the first principle of thermodynamics $d(\rho V)=-P d V$ obtaining the conservation equation $\dot{\rho}=-3 H(\rho+P)$. Note also that, when $F(\mathcal{R}, \mathcal{I})=\mathcal{R}+\mathcal{I}$ one obtains the usual Friedmann equation

$$
H^{2}+\frac{k}{a^{2}}=\frac{\rho}{3 M_{p l}^{2}} .
$$

Finally, dealing with the flat FLRW geometry, since $\mathcal{R}=0$, we will use the notation $f(\mathcal{I}) \equiv F(0, \mathcal{I})$. Then, given a curve $g(\mathcal{I})=\frac{2 \rho}{M_{p l}^{2}}$ in the plane $(\mathcal{I}, \rho)$ we can obtain the corresponding $f$ theory solving the first order differential equation (12), where $\rho$ must be replaced by $g(\mathcal{I})$. Using the variation of constants method for first order differential equations, the result is given by:

$$
f(\mathcal{I})=-\frac{\sqrt{-\mathcal{I}}}{2} \int \frac{g(\mathcal{I})}{\mathcal{I} \sqrt{-\mathcal{I}}} d \mathcal{I}
$$

\subsection{Mimicking holonomy corrected Loop Quantum Cosmology}

Dealing with the flat FLRW geometry with $N=1$, i.e., working, as usual, in co-moving coordinates, one has $\mathcal{R}=0$. Then, redefining the Hubble parameter as $\bar{H}=\sqrt{3 \rho_{c}} M_{p l} H$ where $\rho_{c}$ is a constant with units of energy density, in order that $\bar{H}$ has the same units as $\rho$, in the plane $(\bar{H}, \rho)$, the simplest closed curve is a circle, and since the energy density has to be positive and the Hubble parameter has to be zero when the energy density vanishes, we must choose a circle centered at $\left(0, \frac{\rho_{c}}{2}\right)$ with radius $\frac{\rho_{c}}{2}$, that is, $\bar{H}^{2}+\left(\rho-\frac{\rho_{c}}{2}\right)^{2}=\frac{\rho_{c}^{2}}{4}$, which in the plane $(H, \rho)$ depicts the well-known ellipse (see Fig. 1) that characterizes the holonomy corrected Friedmann equation in Loop Quantum Cosmology (LQC) (see for instance [27, 28, 86])

$$
\frac{H^{2}}{\frac{\rho_{c}}{12 M_{p l}^{2}}}+\frac{\left(\rho-\frac{\rho_{c}}{2}\right)^{2}}{\frac{\rho_{c}^{2}}{4}}=1 \Longleftrightarrow H^{2}=\frac{\rho}{3 M_{p l}^{2}}\left(1-\frac{\rho}{\rho_{c}}\right)
$$

The meaning of $\rho_{c}$, which is called the critical density, is the maximum value of the energy density. Since this equation could be written as

$$
\rho=\frac{\rho_{c}}{2}\left(1 \pm \sqrt{1+\frac{2 \mathcal{I} M_{p l}^{2}}{\rho_{c}}}\right)
$$

where the sign - correspond to the lower branch of the ellipse, i.e., to values of $\rho$ between 0 and $\rho_{c} / 2$ and the sign + to the upper one, that is, to values between $\rho_{c} / 2$ and $\rho_{c}$, one can see that the energy density is bi-valued as a function of $\mathcal{I}$, which, taking into account that mathematically the square root is bi-valued, we will write as

$$
\rho=\frac{\rho_{c}}{2}\left(1-\sqrt{1+\frac{2 \mathcal{I} M_{p l}^{2}}{\rho_{c}}}\right)
$$

where we use the convention that the sign positive of the square root corresponds to the lower branch and the negative to the upper one, i.e., $\rho=\frac{\rho_{c}}{2}\left(1-\sqrt{1+\frac{2 \mathcal{I} M_{p l}^{2}}{\rho_{c}}}\right)$ in the lower branch and $\rho=\frac{\rho_{c}}{2}\left(1+\sqrt{1+\frac{2 \mathcal{I} M_{p l}^{2}}{\rho_{c}}}\right)$ in the upper
one. 


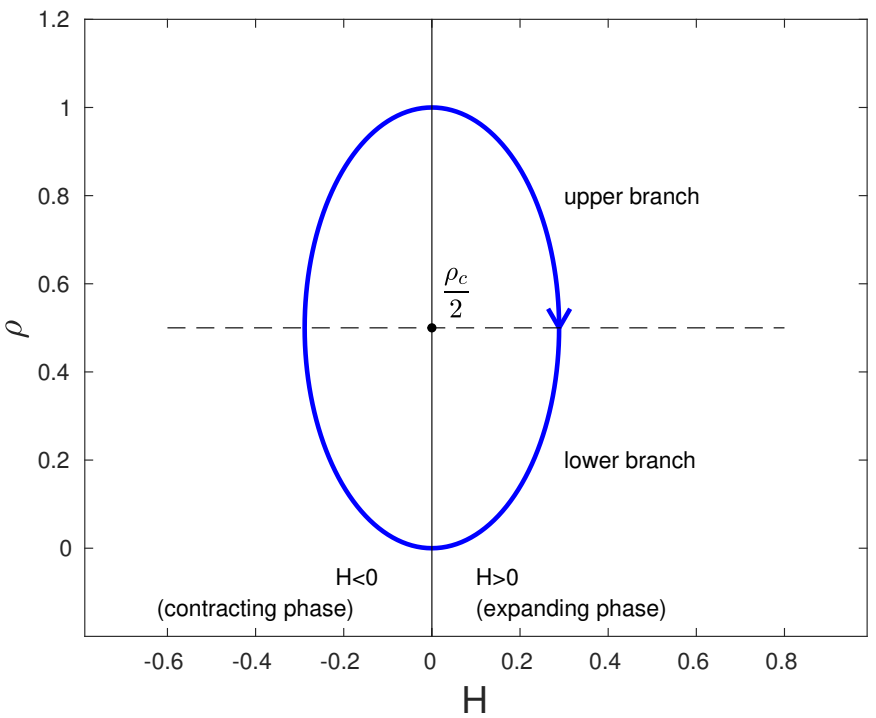

(a) Hamiltonian constraint or equivalently holonomy corrected Friedmann equation in LQC (units: $\rho_{c}=M_{P l}=1$ ).

The arrow shows that the dynamics is ant-clockwise for non-phantom matter.

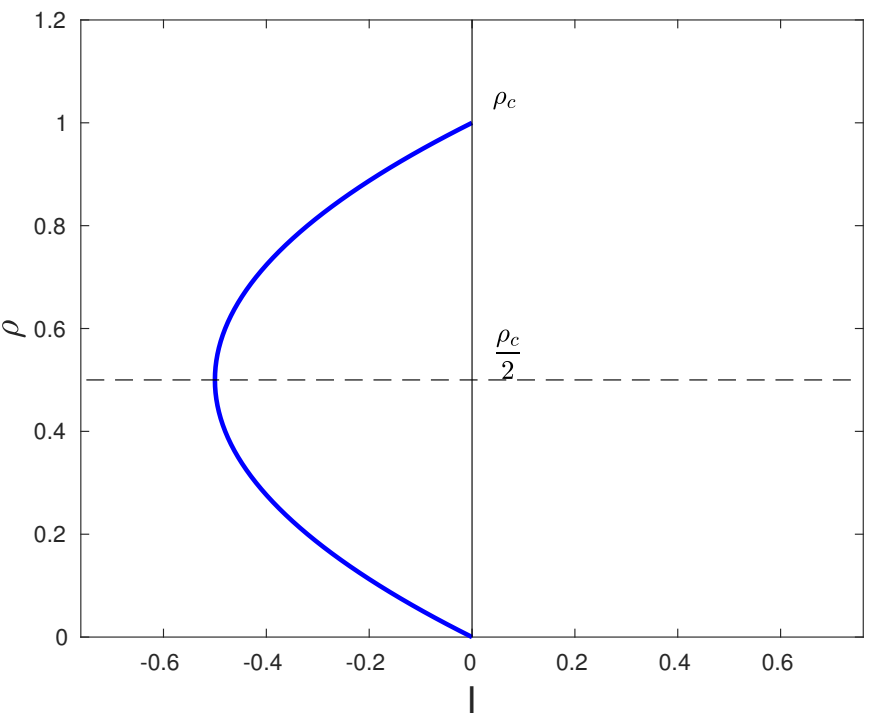

(b) Hamiltonian constraint in the plane $(\mathcal{I}, \rho)$.

FIG. 1: Curve depicting the holonomy corrected Friedmann equation.

Then, to depict this constraint we will need bi-valued functions $f$, which could easily be obtained integrating the equation (14), [51,53, 86]:

$$
f(\mathcal{I})=\frac{\rho_{c}}{M_{p l}^{2}}\left(1-\sqrt{1-s^{2}}-s \arcsin s\right)
$$

where $s \equiv \sqrt{-\frac{2 \mathcal{I} M_{p l}^{2}}{\rho_{c}}}=\sqrt{\frac{12 H^{2} M_{p l}^{2}}{\rho_{c}}}$, belongs in the interval $[0,1]$.

Now one has to decide how one choose the values of $f$ in the upper and lower branch. Here, we take two different prescriptions to define the bi-valued function whose picture appears in Fig. 2:

1. Prescription 1: We choose the sign of the square root positive in the lower branch and negative in the upper one and, $\arcsin s \equiv \int_{0}^{s} \frac{1}{\sqrt{1-\bar{s}^{2}}} d \bar{s}$ where the criterium for the sign of the square root is the same.

2. Prescription 2: We choose the sign of the square root positive in the lower branch and negative in the upper one and, $\arcsin s \equiv \int_{0}^{s} \frac{1}{\sqrt{1-\bar{s}^{2}}} d \bar{s}$ in the lower branch and $\arcsin s \equiv \int_{0}^{s} \frac{1}{\sqrt{1-\bar{s}^{2}}} d \bar{s}+\pi$ in the upper one, with the same criterium for the sign of the square root.

The advantage of the second prescription is that it ensures that the function $f$ is uni-valued when both branches match, i.e. at $s=1$, and in this prescription the function $\arcsin s$ is always positive due to the $\pi$ added in upper branch. Moreover, as we will show dealing with perturbations, the square of the velocity of sound changes from one prescription to the other.

However, in spite of the authors of [51, 52, 86] arguing that the equation $(18)$ gives the $f$ theory that mimics LQC, they only use $f$ as a one-valued function, meaning that they are only considering the lower branch of the ellipse. We insist in this essential point, to obtain the equivalent background to LQC one needs a bi-valued function.

Some important final remarks are in order:

1. As has been pointed out in [90, the bi-valued functions $\sqrt{1-s^{2}}$ and $\arcsin s$ could be avoided, allowing the variable $s$ to belongs in the interval $[-1,1]$ (note that by definition it belongs in $[0,1]$ ) and performing the transformation $s=\sin (2 \psi)$ with $\psi \in[0, \pi]$. Then, the ellipse is parametrized in the following way

$$
H=\sqrt{\frac{\rho_{c}}{12 M_{p l}^{2}}} \sin (2 \psi), \quad \rho=\rho_{c} \sin ^{2} \psi,
$$




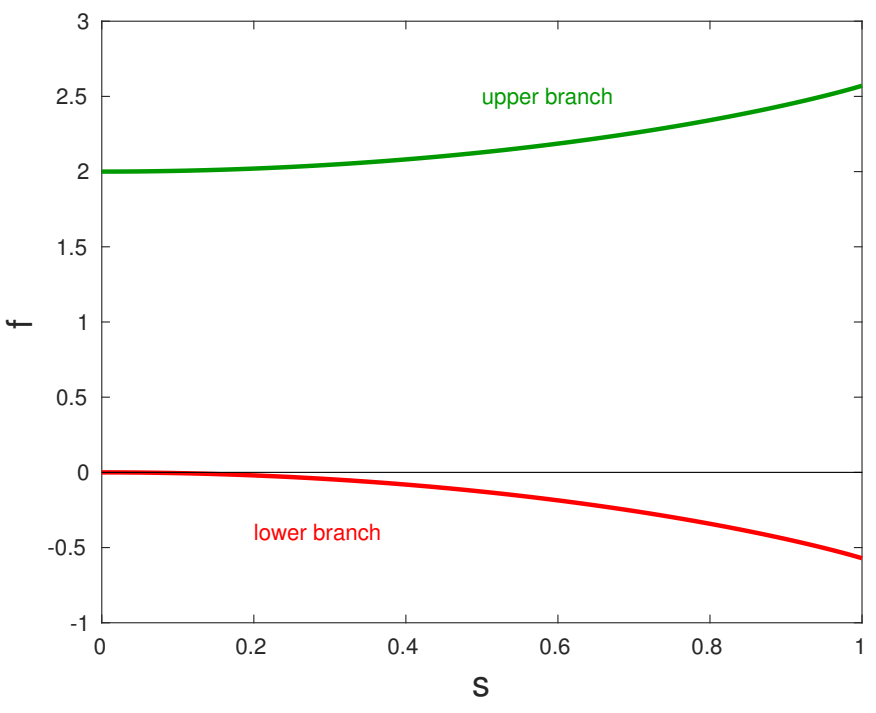

(a) Prescription 1 .

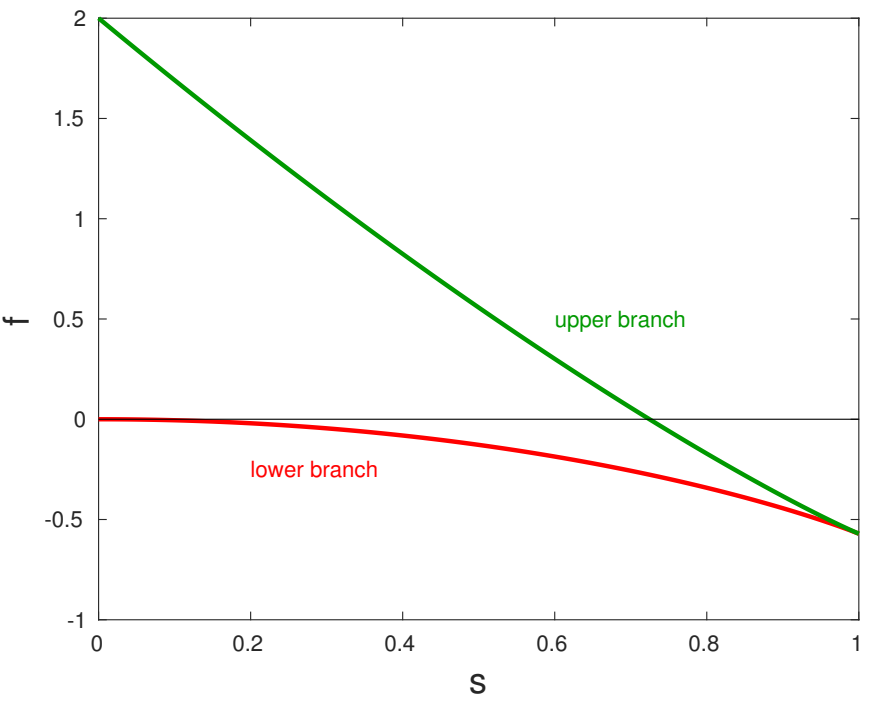

(b) Prescription 2.

FIG. 2: Bivalued function $f$ for the upper (green) and lower (red) branches.

and the total Hamiltoninan

$$
\mathcal{H}_{t o t} \equiv \dot{V} p_{V}+\dot{\phi} p_{\phi}-\mathcal{L}_{t o t}=-\frac{\rho_{c}}{M_{p l}^{2}}\left(1-\sqrt{1-s^{2}}\right)+\rho,
$$

where $p_{V}=\frac{\partial \mathcal{L}_{t o t}}{\partial \dot{V}}$ and $p_{\phi}=\frac{\partial \mathcal{L}_{t o t}}{\partial \dot{\phi}}$ becomes

$$
\mathcal{H}_{t o t}=-\frac{\rho_{c}}{M_{p l}^{2}} \sin ^{2} \psi+\rho,
$$

which coincides with the usual Hamiltonian in LQC.

2. At low energy densities a viable theory must coincide with General Relativity, so in the flat FLRW geometry it will be $H^{2}=\frac{\rho}{3 M_{p l}^{2}}$, meaning that for $H=0$ the energy density vanishes. On the other hand, if one has a bounce, at the bounce the energy density does not vanish when the Hubble parameter is zero. This means that, in a theory with bounces where the modified Friedmann equation is a constraint relating only $H$ and $\rho$, the energy density as a function of the Hubble parameter has to be a multi-valued function, and thus, multi-valued functions $f$ are needed to obtain the corresponding modified Friedmann equation.

3. Bouncing cosmologies mimicking LQC could also be obtained, in a gauge invariant, approach named $F(R, T)$ gravity [87, where $T$ denotes the trace of the the stress tensor. In this theory, on the contrary of the one proposed in this manuscript, it is the matter sector which is modified. Dealing with the particular case $F(R, T)=$ $R+\frac{1}{M_{p l}^{2}} f(T)$, the modified Friedmann equation, for co-moving observers in the flat FLRW spacetime, becomes 88

$$
3 H^{2}=\frac{1}{M_{p l}^{2}}\left[\left(1+f^{\prime}(T)\right) \rho+f^{\prime}(T) \mathcal{L}_{m}-\frac{f(T)}{2}\right]
$$

Then, assuming that the universe is filled by an scalar field (this is necessary in order to deal with primordial perturbations) on has $\mathcal{L}_{m}=P$, but in order that the right hand side of 22 only depends of $T$, one has to assume that the field mimics a perfect fluid with a constant Equation of State parameter $w \equiv \frac{P}{\rho}$. To simplify, we assume that the scalar field mimics a dust fluid $(w=0 \Longrightarrow T=-\rho)$, obtaining the equation

$$
3 H^{2}=-\frac{1}{M_{p l}^{2}}\left[\left(1+f^{\prime}(T)\right) T+\frac{f(T)}{2}\right] .
$$


To obtain the uni-valued function $f$ leading to the same Friedmann equation as in holonomy corrected LQC, one only has to solve the first order differential equation

$$
\left(1+f^{\prime}(T)\right) T+\frac{f(T)}{2}=T\left(1+\frac{T}{\rho_{c}}\right)
$$

whose solution is $f(T)=\frac{2}{5 \rho_{c}} T^{2}$.

Unfortunately, apart from other difficulties that leads this approach unviable [89], the continuity equation differs form the usual one, namely $\dot{\rho}=-3 H \rho$ for a pressureless fluid. For $f(T)=\frac{2}{5 \rho_{c}} T^{2}$, one has

$$
\dot{\rho}=-\frac{3 H \rho}{1-\frac{2 \rho}{\rho_{c}}}\left(1-\frac{4 \rho}{5 \rho_{c}}\right)
$$

which is singular at $\rho=\rho_{c} / 2$, i.e., when both branches of the ellipse matches. So, only few unnatural an unrealistic bouncing solutions could be obtained (see Section 4 of [88]).

4. Recently, the function (18) has been used in the context of mimetic gravity 90 92 and also as a function of the Carminati-MacLenaghan scalars [93, to reproduce, in a covariant way, the same background as in holonomy corrected LQC. A perturbed theory of this approach has not been devoloped yet, although it deserve future investigation.

\subsection{Gravitational equations in the $F$ theory}

To get the gravitational equation we start with the total action

$$
S_{F, t o t}=\int_{t_{1}}^{t_{2}}\left\{\int_{\Sigma_{t}} N \sqrt{\gamma}\left(\frac{M_{p l}^{2}}{2} F(\mathcal{R}, \mathcal{I})+\mathcal{L}_{\text {matt }}\right) d^{3} x\right\} d t
$$

where we assume the universe is filled by an scalar field minimally coupled with gravity, whose Lagrangian is

$$
\mathcal{L}_{\text {matt }}=\left(-\frac{\phi_{\mu} \phi^{\mu}}{2}-V(\phi)\right)=\left(\frac{\dot{\phi}^{2}}{2 N^{2}}-\frac{N^{i}}{N^{2}} \dot{\phi} \phi_{i}-\frac{1}{2}\left(\gamma^{i j}-\frac{N^{i} N^{j}}{N^{2}}\right) \phi_{i} \phi_{j}-V(\phi)\right) .
$$

Then, to obtain the constraints and dynamical equations we have to perform the variation with respect the variables $N, N_{i}, \gamma_{i j}$ and with respect the scalar field $\phi$.

Taking into account that the components of the extrinsic curvature could be written as follows 67

$$
K_{i j}=\frac{1}{2 N}\left(D_{i} N_{j}+D_{j} N_{i}-\dot{\gamma}_{i j}\right),
$$

where $D$ is the induced Levi-Civita connection in the space tangent of $\Sigma_{t}$, that the variation of the intrinsic curvature is

$$
\delta \mathcal{R}=\mathcal{R}_{i j} \delta \gamma^{i j}+D_{k}\left(\gamma^{i j} \delta \Gamma_{i j}^{k}-\gamma^{k j} \delta \Gamma_{i j}^{i}\right)
$$

where $\mathcal{R}_{i j}=\mathcal{R} i c\left(\partial_{i}, \partial_{j}\right)$ are the entries of the Ricci tensor and $\Gamma_{i j}^{k}$ are the Christoffel symbols, and using the formula

$$
\gamma^{k m}\left(D_{i} \delta \gamma_{m j}+D_{j} \delta \gamma_{m i}-D_{m} \delta \gamma_{i j}\right)=2 \delta \Gamma_{i j}^{k}
$$

and the Gauss divergence theorem one gets (see 94, to our knowledge the first instance where these equations were derived):

$$
\begin{gathered}
F-2 \mathcal{I} F_{\mathcal{I}}=-\frac{2}{M_{p l}^{2}} \partial_{N}\left(N \mathcal{L}_{\text {matt }}\right) \\
D_{j}\left(F_{\mathcal{I}} \pi_{i}^{j}\right)=-\frac{N}{M_{p l}^{2}} \partial_{N^{i}} \mathcal{L}_{m a t t}
\end{gathered}
$$




$$
\begin{gathered}
-\left(D^{j} D_{i}-\delta_{i}^{j} D^{2}\right)\left(F_{\mathcal{R}} N\right)+N G_{F, i}^{j}+2 N F_{\mathcal{I}}\left(K^{m j} K_{i m}-T r(K) K_{i}^{j}\right) \\
-\frac{1}{\sqrt{\gamma}} \gamma_{k i} \partial_{t}\left(\sqrt{\gamma} F_{\mathcal{I}} \pi^{k j}\right)-D_{k}\left(F_{\mathcal{I}}\left(\pi_{i}^{k} N^{j}+\pi^{k j} N_{i}-\pi_{i}^{j} N^{k}\right)\right)=\frac{N}{M_{p l}^{2}} T_{i}^{j},
\end{gathered}
$$

where $\pi_{i}^{j}$ are the entries of the tensor $\pi \equiv K-\operatorname{Tr}(K) \gamma, D^{2}=D^{k} D_{k}$ is the three-dimensional Laplace-Beltrami operator, $G_{F}=-\frac{1}{2} F \gamma+F_{\mathcal{R}} \mathcal{R} i c$ is the three-dimensional modified Einstein tensor, and $T_{i}^{j}=\frac{2}{\sqrt{\gamma}} \gamma^{m j} \partial_{\gamma_{i m}}\left(\sqrt{\gamma} \mathcal{L}_{\text {matt }}\right)$ are the entries of the three-dimensional stress-energy tensor.

The equations (31) and (32) are the well-known Hamiltonian and diffeomorphism constraints, and the equation $(33)$ is the dynamical one.

For the scalar field, the conservation equation is $-\nabla^{2} \phi+V_{\phi}=0$, where $\nabla^{2}=\nabla^{i} \nabla_{i}$ is the Laplace-Beltrami operator in the full manifold $\mathcal{M}$, which in the ADM formalism becomes

$$
\frac{1}{\sqrt{\gamma} N} \partial_{t}\left(\frac{\sqrt{\gamma}}{N} \dot{\phi}\right)-\frac{1}{N} D_{i}\left(N \phi^{i}\right)-\frac{1}{N} D_{i}\left(\frac{N^{i}}{N}\left(\dot{\phi}-N^{j} \phi_{j}\right)\right)+V_{\phi}=0
$$

Finally, once these equations are obtained one can deal with the Cauchy initial value problem as in GR, i.e., given a fixed foliation, in our case the preferred one, one has to choose a slice at some time $t_{0}$, namely $\Sigma$, which is assumed to be a Cauchy hyper-surface), and we have to consider on it the set of variables $(\gamma, K, \phi, \dot{\phi})$ where $\gamma$ is the metric and $K$ the extrinsic curvature tensor on $\Sigma$, which has to satisfy the Hamiltonian and Diffeomorphism constraints. Then, to solve the dynamical equations one has to follow, as in GR, some of the schemes proposed in numerical relativity (see 95,96 for reviews).

\section{SCALAR PERTURBATIONS}

In the previous Section we have shown the lack of covariance of our approach. Nevertheless, our theory is gauge invariant at the perturbation level. To prove it, we consider the general line element containing scalar perturbations [75]

$$
d s^{2}=-(1+2 \Psi) d t^{2}+2 a \partial_{i} B d t d x^{i}+a^{2}\left[(1-2 \Phi) \delta_{i j}-2 \partial_{i j}^{2} E-h_{i j}\right] d x^{i} d x^{j}
$$

where $h$ is a symmetric, traceless and transverse tensor: $h_{i}^{i}=\partial_{i} h^{i j}=0$, and $\Psi, \Phi$ and $E$ are scalars [83].

In order to deal with scalar perturbations, since there are two degrees of freedom we choose the so-called Newtonian or Longitudinal gauge, where the two metric variables $E$ and $B$ vanishes. Then, we obtain the metric

$$
d s^{2}=-(1+2 \Psi) d t^{2}+a^{2}(1-2 \Phi) \delta_{i j} d x^{i} d x^{j} .
$$

Remark 3.1 In General Relativity, i.e., when $F(\mathcal{R}, \mathcal{I})=\mathcal{R}+\mathcal{I}$ and the matter part of the Lagrangian is depicted by an scalar field, one has $\Phi=\Psi$ [83], but in general, for a $F$ theory, these functions are different (see section 7 of [97]). Fortunately, as we will immediately show, if one chooses $F(\mathcal{R}, \mathcal{I})=\mathcal{R}+f(\mathcal{I})$ the equality holds. Due to this simplification in the theory, we will only consider this kind of $F$ models.

Since in this gauge one has $N^{2}=(1+2 \Psi), \gamma_{i j}=a^{2}(1-2 \Phi) \delta_{i j}$ and $N^{i}=0$, the extrinsic curvature is given by

$$
\mathcal{I}=-\frac{6(H(1-2 \Phi)-\dot{\Phi})^{2}}{(1-2 \Phi)^{2}(1+2 \Psi)} \approx-6 H^{2}+12 H(\Psi H+\dot{\Phi})
$$

where the symbol $\approx$ means: up to first order.

On the other hand, since the Christoffel symbols satisfy

$$
\Gamma_{i j}^{k}=-\frac{1}{1-2 \Phi}\left(\delta_{k i} \Phi_{j}+\delta_{k j} \Phi_{i}-\delta_{i j} \delta^{m k} \Phi_{m}\right),
$$


the entries of the Ricci tensor will be

$$
\mathcal{R}_{i j}=\partial_{k} \Gamma_{i j}^{k}-\partial_{j} \Gamma_{i k}^{k}+\Gamma_{i j}^{k} \Gamma_{k l}^{l}-\Gamma_{i k}^{l} \Gamma_{l j}^{k} \approx \partial_{k} \Gamma_{i j}^{k}-\partial_{j} \Gamma_{i k}^{k}=\Phi_{i j}+\delta_{i j} \Delta \Phi,
$$

and the scalar curvature will become $\mathcal{R} \approx \frac{4}{a^{2}} \Delta \Phi$, where $\Delta=\sum_{i=1}^{3} \partial_{i i}^{2}$ is the standard three-dimensional Laplacian.

Now, in the linear approximation, we will use the following notation:

$$
\begin{gathered}
\mathcal{I}=\mathcal{I}_{0}+\mathcal{I}_{1}, \quad \text { with } \quad \mathcal{I}_{0}=-6 H^{2}, \quad \mathcal{I}_{1}=12 H(\Psi H+\dot{\Phi}), \\
\phi=\phi_{0}(t)+\delta \phi \Longrightarrow V(\phi)=V_{0}+V_{\phi, 0} \delta \phi, \quad \text { with } \quad V_{0}=V\left(\phi_{0}\right), \quad V_{\phi, 0}=V_{\phi}\left(\phi_{0}\right) . \\
g=f-2 \mathcal{I} f_{\mathcal{I}}, \quad g_{0}=g\left(\mathcal{I}_{0}\right) \quad \text { and } \quad g_{\mathcal{I}, 0}=\partial_{\mathcal{I}} g\left(\mathcal{I}_{0}\right) \equiv g_{\mathcal{I}}\left(\mathcal{I}_{0}\right),
\end{gathered}
$$

where the meaning of $g$ is that the equation $g_{0}=\frac{2 \rho_{0}}{M_{p l}^{2}}$, where $\rho_{0}$ is the non-perturbed energy density, is the nonperturbed hamiltonian constraint, i.e., the modified Friedmann equation.

For $i \neq j$, the equation 33 becomes

$$
-D^{j} D_{i}\left(F_{\mathcal{R}} N\right)+N G_{F, i}^{j}=0,
$$

which for our choice $F(\mathcal{R}, \mathcal{I})=\mathcal{R}+f(\mathcal{I})$ (essential to obtain the desired result), becomes

$$
-D^{j} D_{i}(N)+N \mathcal{R}_{i}^{j}=0 \Longrightarrow \partial_{i j}^{2}(\Phi-\Psi)=0,
$$

which leads to the solution $\Phi=\Psi$. Then, the perturbed equations become:

$$
\begin{gathered}
\frac{1}{a^{2}} \Delta \Phi+3 H^{2} g_{\mathcal{I}, 0} \Phi+3 H g_{\mathcal{I}, 0} \dot{\Phi}=\frac{1}{2 M_{p l}^{2}}\left(\dot{\phi}_{0}\left(\dot{\delta \phi}-\dot{\phi}_{0} \Phi\right)+V_{\phi, 0} \delta \phi\right), \\
-g_{\mathcal{I}, 0}(H \Phi+\dot{\Phi})=\frac{1}{2 M_{p l}^{2}} \dot{\phi}_{0} \delta \phi \\
-g_{\mathcal{I}, 0} \ddot{\Phi}-\left(4 H g_{\mathcal{I}, 0}+\dot{g}_{\mathcal{I}, 0}\right) \dot{\Phi}-\left(\left(3 H^{2}+2 \dot{H}\right) g_{\mathcal{I}, 0}+H \dot{g}_{\mathcal{I}, 0}\right) \Phi=\frac{1}{2 M_{p l}^{2}}\left(\dot{\phi}_{0}\left(\dot{\delta \phi}-\dot{\phi}_{0} \Phi\right)-V_{\phi, 0} \delta \phi\right) .
\end{gathered}
$$

Combining the equations and using the cosmic time one gets the dynamical equation for the Newtonian potential the following equation, which coincides with the one of GR (see equation (6.48) of [97]) when one takes $f(\mathcal{I})=\mathcal{I}$,

$$
\Phi^{\prime \prime}-c_{S}^{2} \Delta \Phi+2\left(\mathcal{H}-\left(\frac{\phi_{0}^{\prime \prime}}{\phi_{0}^{\prime}}+\epsilon\right)\right) \Phi^{\prime}+2\left(\mathcal{H}^{\prime}-\mathcal{H}\left(\frac{\phi_{0}^{\prime \prime}}{\phi_{0}^{\prime}}+\epsilon\right)\right) \Phi=0
$$

where the square of the velocity of sound for scalar perturbations is $c_{S}^{2}=-\frac{1}{g_{\mathcal{I}, 0}}$ and we have introduced the notation $\epsilon=-\frac{g_{\mathcal{I}, 0}^{\prime}}{2 g_{\mathcal{I}, 0}}$.

Now introducing, as in standard cosmology, (see for example formulae (8.56)-(8.58) of [83]) the gauge invariant Mukhanov-Sasaki (M-S) variables

$$
v_{S}=a\left(\delta \phi+\frac{\phi_{0}^{\prime}}{\mathcal{H}} \Phi\right), \quad z_{S}=\frac{a \phi_{0}^{\prime}}{\mathcal{H}},
$$

the equation 48 becomes, in the Fourier space, the following gauge invariant M-S equation

$$
v_{S, k}^{\prime \prime}+\left(c_{S}^{2} k^{2}-\frac{z_{S}^{\prime \prime}}{z_{S}}\right) v_{S, k}=0
$$

Some important final remarks are in order: 
1. If one changes $\mathcal{I}$ by $\mathcal{T}$, where $\mathcal{T}$ is the torsion that appears when one uses the Weitzenböck connection instead of the Levi-Civita one, these equations are practically the same as the ones that appear in teleparallel $f(\mathcal{T})$ gravity [59]. The difference appears in equation $(45)$, where in $f(\mathcal{T})$ gravity the coefficient that multiplies the Laplacian is $\frac{f_{\mathcal{T}}}{a^{2}}$, and it is very important because the velocity of sound changes from one theory to the other. Effectively, in this theory the equation of the Newtonian potential is the same as (48) but with a velocity of sound given by $\tilde{c}_{S, s}^{2}=-\frac{f_{\mathcal{I}, 0}}{g_{\mathcal{I}, 0}}$. Moreover, in teleparallel $f(\mathcal{T})$ gravity the variables $v_{S}$ and $z_{S}$ has to be 61 , 62

$$
v_{S}=\frac{a}{\sqrt{\left|f_{\mathcal{I}, 0}\right|}}\left(\delta \varphi+\frac{\phi_{0}^{\prime}}{\mathcal{H}} \Phi\right), \quad z_{S}=\frac{a \phi_{0}^{\prime}}{\sqrt{\left|f_{\mathcal{I}, 0}\right|} \mathcal{H}}
$$

in order to recover the M-S equation 50 , but replacing $c_{S}^{2}$ by $\tilde{c}_{S}^{2}$.

2. Applying the theory to the particular model given in 18 , we will obtain a theory, which we name Extrinsic curvature $L Q C$, where $-g_{\mathcal{I}, 0}=\frac{1}{\Omega}$, with $\Omega \equiv 1-\frac{2 \rho}{\rho_{c}}$, and thus, $c_{S}^{2}=\Omega$ and $\epsilon=\frac{\Omega^{\prime}}{2 \Omega}$. Then, the equations 48 and (50) are identical to the ones obtained in holonomy corrected LQC [47, which from our point of view supports the consistency of our approach to obtain bouncing cosmologies via modified gravity in the ADM formalism.

3. On the contrary, in teleparallel LQC, since

$$
f_{\mathcal{I}, 0}=\frac{1}{s} \arcsin s
$$

where $s=\sqrt{\frac{-2 \mathcal{I} M_{p l}^{2}}{\rho_{c}}}$, the square of the velocity of the sound will be $\tilde{c}_{S}^{2}=\frac{\Omega}{s}$ arcsin $s$, and contrary to holonomy corrected and extrinsic curvature LQC, is always positive if one takes the first prescription below formula (18), but taking the second one, $\tilde{c}_{S}^{2}$ is positive in the lower branch, negative in the upper one and unbounded at the bounce.

\section{TENSOR PERTURBATIONS}

For metric perturbations the line element, in the preferred foliation, is

$$
d s^{2}=-d t^{2}+a^{2}\left(\delta_{i j}-h_{i j}\right) d x^{i} d x^{j}
$$

where, as we have already introduced in the previous section, $h$ is a symmetric, traceless and transverse tensor: $h_{i}^{i}=\partial_{i} h^{i j}=0$. Due to these properties one has $\sqrt{\gamma} \approx a^{3}, \operatorname{Tr}(K) \approx-3 H$ and $\mathcal{I} \cong-6 H^{2}$. Then, the equation 33 reduces to

$$
2 f_{\mathcal{I}, 0}\left(K^{m j} K_{m i}+3 H K_{i}^{j}\right)+G_{F, i}^{j}-\frac{1}{a^{3}} \gamma_{k i} \partial_{t}\left(a^{3} f_{\mathcal{I}, 0} \pi^{k j}\right)=0
$$

And taking into account that $\mathcal{R}_{i}^{j} \approx-\frac{1}{a^{2}} \Delta h_{i}^{j}$ and $\mathcal{R} \approx 0$, the equation for the gravitational waves becomes

$$
f_{\mathcal{I}, 0} \ddot{h}_{i}^{j}+3 H f_{\mathcal{I}, 0} \dot{h}_{i}^{j}-\frac{1}{a^{2}} \Delta h_{i}^{j}+\dot{f}_{\mathcal{I}, 0} \dot{h}_{i}^{j}=0 .
$$

On can see that, this equation differs from the one obtained in $f(\mathcal{T})$ gravity, in the coefficient that multiplies the Laplacian.

Introducing the variable $v_{T}=h z_{T}$ with $z_{T}=a M_{p l} \sqrt{\left|f_{\mathcal{I}, 0}\right|}$ and $h$ denoting $h_{i}^{j}$, one obtains, in Fourier space, the M-S equation for tensor perturbations

$$
v_{T, k}^{\prime \prime}+\left(c_{T}^{2} k^{2}-\frac{z_{T}^{\prime \prime}}{z_{T}}\right) v_{T, k}=0,
$$

where now the square of the velocity of the sound is equal to $c_{T}^{2}=\frac{1}{f_{\mathcal{I}, 0}}$.

The variables $v, z_{T}$ are the same used en teleparallel $f(\mathcal{T})$ gravity, and the M-S for gravitational waves only differs in the square of the velocity of sound, which in teleparallelism is always 1. 
Now, if we consider the function that leads to the same background as holonomy corrected LQC, one has $f_{\mathcal{I}, 0}=$ $\frac{1}{s} \arcsin s$, what implies that, if one takes the first prescription below $(18)$ the square of the velocity of the sound $c_{T}^{2}=\frac{1}{f_{\mathcal{I}, 0}}$ is positive in the lower branch of the ellipse and negative and the upper one, as in holonomy corrected LQC, although it is different because in LQC for both kind of perturbations, scalar and tensors, one has $c_{T}^{2}=\Omega$. Moreover, in extrinsic curvature LQC following this prescription, the square of the velocity is discontinuous when the branches match. This does not happen if one chooses the second prescription where $c_{T}^{2}$ is always positive and continuous.

However, the main difference with holonomy corrected LQC appears in the definition of $z_{T}$, because in the former case one has [48] $z_{T}=\frac{a M_{p l}}{\sqrt{c_{T}^{2}}}=\frac{a M_{p l}}{\sqrt{\Omega}}$, which becomes complex in the upper branch, being an exotic feature of holonomy corrected LQC, and reducing the ratio of tensor to scalar perturbations to negligible values in the matter bounce scenario (see next section) [62, 98. This does not happen in theories such as GR, $F(R)$ or $F(\mathcal{T})$ gravity, and neither does it in our approach defining $z_{T}=\frac{a M_{p l}}{\sqrt{\left|c_{T}^{2}\right|}}=a M_{p l} \sqrt{\frac{|\arcsin s|}{s}}$. But, if one chooses $z_{T}=\frac{a M_{p l}}{\sqrt{c_{T}^{2}}}=a M_{p l} \sqrt{\frac{\arcsin s}{s}}$ (which could be done because the equation (56) is the same for both definitions of $z_{T}$ ), for the first prescription of $f$, since the square of the velocity of sound becomes negative in the upper branch, $z_{T}$ will be complex in the upper branch as in holonomy corrected LQC. This will reduce the tensor/scalar ratio to negligible values in the case of the matter bounce scenario, which does not happen with the second prescription, because in that case $c_{T}^{2}=\frac{s}{\arcsin s}$ is always positive.

Summing up, the M-S for scalar and tensor perturbation in Extrinsic curvature $L Q C$ are:

$$
v_{S, k}^{\prime \prime}+\left(c_{S}^{2} k^{2}-\frac{z_{S}^{\prime \prime}}{z_{S}}\right) v_{S, k}=0, \quad v_{T, k}^{\prime \prime}+\left(c_{T}^{2} k^{2}-\frac{z_{T}^{\prime \prime}}{z_{T}}\right) v_{T, k}=0,
$$

where, if one considers the model that leads to the same background as holonomy corrected LQC, i.e., given by the function 18 and we use the second prescription, the square of the velocity of sound is $c_{S}^{2}=\Omega=1-\frac{2 \rho}{\rho_{c}}$ for scalar perturbations and $c_{T}^{2}=\frac{s}{\arcsin s}$ for the tensor ones. Then, since in holonomy corrected LQC one has $c_{S}^{2}=c_{T}^{2}=\Omega$ we see that both approaches coincide for scalar perturbations, but for tensor ones they differ in the square of the velocity of sound, where in holonomy corrected LQC the sign of the square of the velocity of sound changes its sign passing from one branch of the ellipse to the other one, but in Extrinsic curvature LQC it is always positive and in fact, for tensor perturbations, as we already explained, our approach is closer to $F(\mathcal{T})$ gravity. In conclusion, Extrinsic curvature LQC leads to the same scalar perturbation equations that holonomy corrected LQC, and very close equations to $F(\mathcal{T})$ gravity for tensor perturbations.

\section{THE MATTER BOUNCE SCENARIO IN THEORIES WITH THE SAME BACKGROUND AS LQC}

In this scenario the universe is filled by dust matter, whose background in LQC, can be mimicked by a scalar field whose potential is 99

$$
V\left(\phi_{0}\right)=2 \rho_{c} \frac{e^{-\frac{\sqrt{3} \phi_{0}}{M_{p l}}}}{\left(1+e^{-\frac{\sqrt{3} \phi_{0}}{M_{p l}}}\right)^{2}},
$$

where $\phi_{0}$ is the homogeneous part of the scalar field, i.e., the unperturbed part. The conservation equation is given by

$$
\ddot{\phi}_{0}+3 H_{ \pm} \dot{\phi}_{0}+V_{\phi_{0}}=0
$$

where $H_{ \pm}= \pm \sqrt{\frac{\rho_{0}}{3 M_{p l}^{2}}\left(1-\frac{\rho_{0}}{\rho_{c}}\right)}$ are the values of the Hubble parameter in the contracting and expanding phase, and $\rho_{0}$ is the unperturbed energy density $\rho_{0}=\frac{\dot{\phi}_{0}^{2}}{2}+V\left(\phi_{0}\right)$. Thus, for each of the infinite solutions of 59 ) one obtains a different background $H(t)$. On the other hand, there is an analytic solution given by

$$
\phi_{0}(t)=\frac{2 M_{p l}}{\sqrt{3}} \ln \left(\sqrt{\frac{3 \rho_{c} t^{2}}{4 M_{p l}^{2}}}+\sqrt{\frac{3 \rho_{c} t^{2}}{4 M_{p l}^{2}}+1}\right),
$$




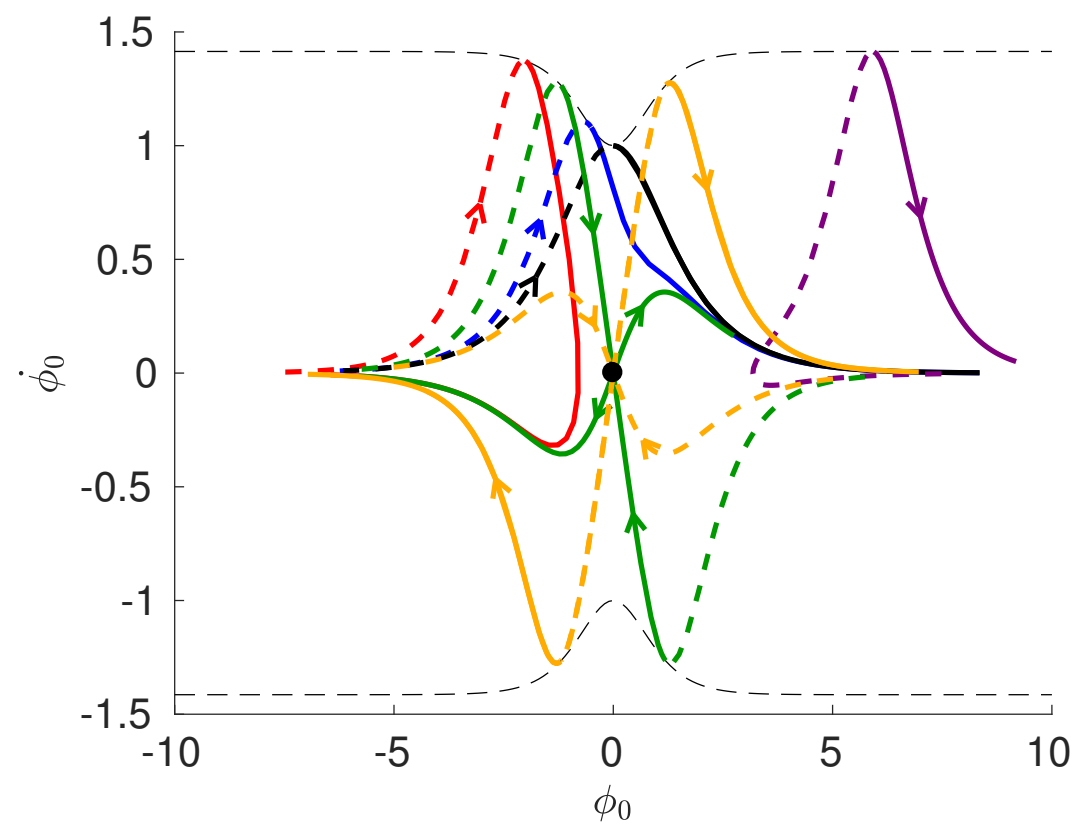

(a) Phase portrait of the system, with curves bouncing from $H<0$ to $H>0$ at the upper bounce curve where $\dot{\phi}_{0}>0$ : the unstable and stable varieties for $H>0$ (green), and $H<0$ (orange) of the critical point at $(0,0)$; orbits bouncing to the left (red) and to the right (blue) of the stable variety; the analytic orbit (black); an orbit bouncing past the unstable variety (purple). Orbits with $H>0$ are plotted as continuous lines; those with $H<0$ as discontinuous lines.

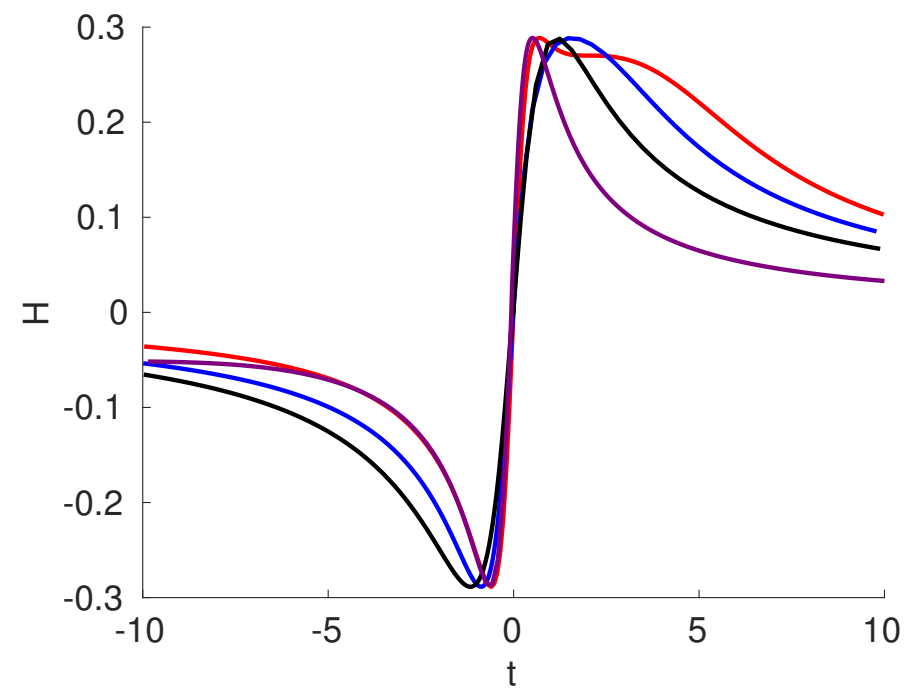

(b) $H(t)$ for the orbits in Fig. 3a bouncing left of the stable variety (red), between the stable and unstable varieties (blue), right of the unstable variety (purple), and for the analytic orbit (black).

FIG. 3: The extrinsic curvature LQC model: possible backgrounds (black: analytic solution; red, blue, purple: qualitatively different alternatives).

which leads to the following analytic background

$$
H(t)=\frac{\frac{\rho_{c} t}{2}}{\frac{3 \rho_{c} t^{2}}{4}+M_{p l}^{2}} .
$$

The other solutions have to be calculated numerically, and lead to different backgrounds (see Figures 3a, 3b, also the figures 8 and 9 of [100] and the corresponding explanation). 
Dealing with the power spectrum, for scalar perturbations it is given by the formula 75 .

$$
P_{S}(k)=\frac{k^{3}}{2 \pi^{2}}\left|\zeta_{k}\right|^{2},
$$

where, as we have already explained in Section III, $\zeta_{k} \equiv \frac{v_{k}}{z_{S}}$ is the curvature fluctuation in co-moving coordinates. In the case of the matter bounce scenario, taking at early times the scale factor $a(t)=\left(\frac{3 \rho_{c} t^{2}}{4 M_{p l}^{2}}\right)^{1 / 3}$, one has $[63]$

$$
P_{S}(k)=\frac{3 \rho_{c}^{2}}{\rho_{p l}}\left(\int_{-\infty}^{\infty} \frac{1}{a(t) z_{S}^{2}(t)} d t\right)^{2}
$$

and for tensor perturbations

$$
P_{T}(k)=\frac{\rho_{c}^{2}}{\rho_{p l}}\left(\int_{-\infty}^{\infty} \frac{1}{a(t) z_{T}^{2}(t)} d t\right)^{2},
$$

where $\rho_{p l} \equiv 64 \pi^{2} M_{p l}^{4}$ is Planck's energy density.

Therefore, the ratio of tensor to scalar perturbations is

$$
r=\frac{\left(\int_{-\infty}^{\infty} \frac{1}{a(t) z_{T}^{2}(t)} d t\right)^{2}}{3\left(\int_{-\infty}^{\infty} \frac{1}{a(t) z_{S}^{2}(t)} d t\right)^{2}}
$$

Using the analytic solution 60 , and the previous notation $\Omega=1-\frac{2 \rho}{\rho_{c}}$ and $s=\sqrt{\frac{12 H^{2} M_{p l}^{2}}{\rho_{c}}}$, one obtains for the first prescription of $f$ :

1. Holonomy corrected LQC: In this case one has $z_{S}=a \frac{\dot{\phi}_{0}}{H}$ and $z_{T}=\frac{a M_{p l}}{\sqrt{\Omega}}$, which leads to $P_{S}(k)=\frac{\pi^{2} \rho_{c}}{9 \rho_{p l}}(\operatorname{see}[98])$ and $P_{T}(k)=0$. Thus, $r=0$.

2. Teleparallel LQC: In this case one has $z_{S}=a \frac{\dot{\phi}_{0}}{H} \sqrt{\frac{s}{|\arcsin s|}}$ and $z_{T}=a M_{p l} \sqrt{\frac{|\arcsin s|}{s}}$, which leads to $P_{S}(k)=$ $\frac{16 C^{2} \rho_{c}}{9 \rho_{p l}}$, where $C=\sum_{n=1}^{\infty} \frac{(-1)^{n}}{(2 n+1)^{2}} \cong 0.91596 \ldots$ is Catalan's constant, and $P_{T}(k)=\frac{16}{3} S i^{2}\left(\frac{\pi}{2}\right) \frac{\rho_{c}}{\rho_{p l}}$ where $S i(x)=$ $\int_{0}^{x} \frac{\sin z}{z} d z$ is the Sine integral function, and thus, $r=3\left(\frac{\left.\operatorname{Si(} \frac{\pi}{2}\right)}{C}\right)^{2} \cong 6.7$.

3. Extrinsic curvature LQC: In this case one has $z_{S}=a \frac{\dot{\phi}_{0}}{H}$ and $z_{T}=a M_{p l} \sqrt{\frac{|\arcsin s|}{s}}$, which leads to $P_{S}(k)=\frac{\pi^{2} \rho_{c}}{9 \rho_{p l}}$ and $P_{T}(k)=\frac{16}{3} S i^{2}\left(\frac{\pi}{2}\right) \frac{\rho_{c}}{\rho_{p l}}$, and thus, $r=\frac{48}{\pi^{2}} S i^{2}\left(\frac{\pi}{2}\right) \cong 9.1$.

We can see that in the case of Teleparallell and Extrinsic curvature LQC, the analytic solution 60 leads to a very high value of the tensor/scalar ratio. Fortunately, as already happens for the case of Teleparallel LQC 62, some backgrounds in Extrinsic curvature LQC lead to a ratio of tensor to scalar perturbations that satisfy the constraint $r \leq 0.12$ provided by the joint analysis of BICEP2/Keck Array and Planck teams [101]. Indeed, the authors' numerical computations, illustrated in Figure 4 show that for backgrounds bouncing at $\frac{\phi_{0}}{M_{p l}} \in[-1.22,-1.20] \cup[1.16,1.20]$ the tensor/scalar ratio $r$ is below 0.12 , while the scalar power spectrum $P_{S}(k)$ is still finite, requiring a fine-tuning of the value of the scalar field at the bounce point. The values of $P_{S}(k)$ in the admissible domain $[-1.22,-1.20] \cup[1.16,1.20]$ grow to infinity abruptly at the domain's upper and lower bounds $\frac{\phi_{0}}{M_{p l}}=-1.22,1.20$, and descend to $16 \frac{\rho_{c}}{\rho_{p l}}$ at the inner bounds $\frac{\phi_{0}}{M_{p l}}=-1.20,1.16$. Consequently, since in Extrinsic curvature LQC, the critical energy density is a parameter of the theory, in order to match with the observational data $P_{S} \cong 2 \times 10^{-9}$ [102] we have to choose $\rho_{c} \sim 10^{-10} \rho_{p l}$ and fine tune the value of the backgrounds at the bouncing time in order to satisfy that $\frac{\phi_{0}}{M_{p l}}$ (at bounce) was extremely close to -1.20 or 1.16 . However in holonomy corrected LQC the critical energy density has a determined value obtained relating the black hole entropy in LQC with the Bekenstein-Hawking entropy formula [103, which is approximately $\rho_{c} \cong 0.4 \rho_{p l}$ disagreeing with the value obtained in the matter bounce scenario.

Finally, note that using the second prescription (see below $(18)$ ) to define the bi-valued function $f$, scalar perturbations in teleparallel LQC are not well defined because the power spectrum of scalar perturbations diverges, this 


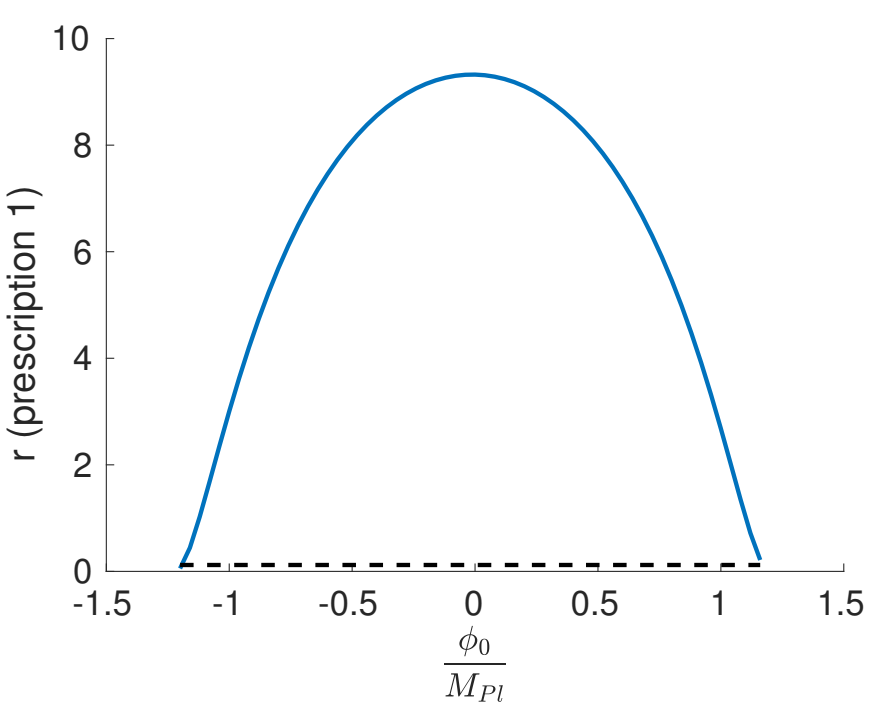

(a) For prescription 1 of $f$.

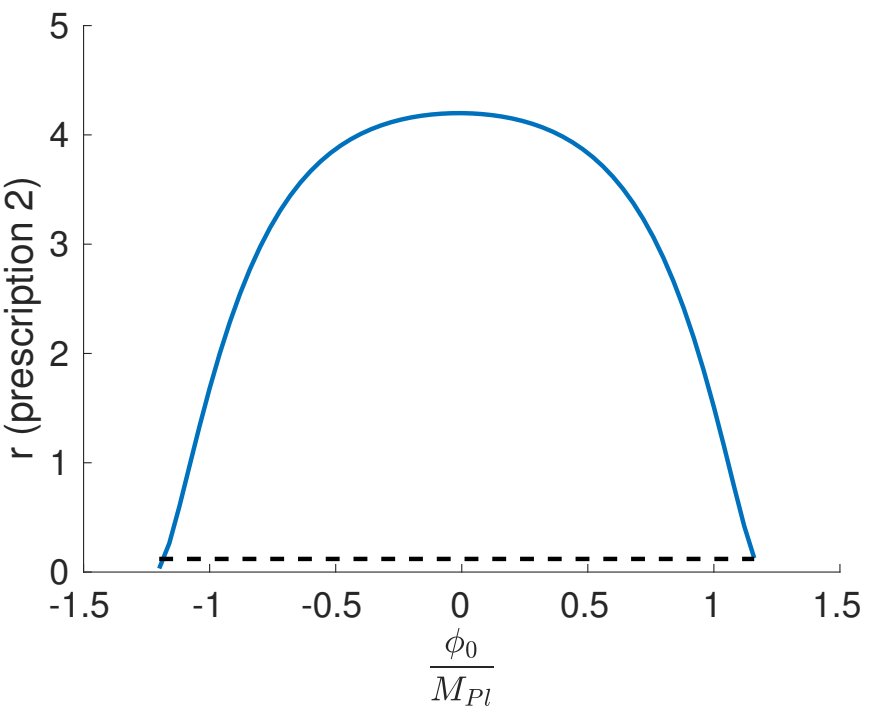

(b) For prescription 2 of $f$.

FIG. 4: Tensor/scalar ratio $r$ for backgrounds solution to Extrinsic curvature LQC Eq. (59). The backgrounds are indexed according to the value of $\phi_{0}$ at bounce. The Planck-BICEP2 bound $r \leq 0.12$ is indicated.

is the reason why the first prescription is used in 62, 63. On the contrary, in extrinsic curvature LQC both kinds of perturbations are well defined: the scalar ones are independent of the prescription and for tensor ones, in both prescriptions, the power spectrum is of the same order. In fact, for the analytic solution 60 we have obtained $P_{T}(k)=\frac{4}{3} S i^{2}(\pi) \frac{\rho_{c}}{\rho_{p l}}$, leading to a tensor/scalar ratio equal to $r=\frac{12}{\pi^{2}} S i^{2}(\pi) \cong 4.1$. Figure 4 illustrates the comparison of the values of the tensor/scalar ratio $r$ for both prescriptions of $f$; the backgrounds for which $r \leq 0.12$ turn out to be almost exactly the same.

\subsection{Improvement of the matter bounce scenario: the matter-ekpyrotic bounce scenario}

As we have seen above when calculating the power spectrum, dealing with the LQC background, it is of the order $\frac{\rho_{c}}{\rho_{p l}}$, then to match with the observational value $P_{S}(k) \sim 2 \times 10^{-9}$, one has to take $\rho_{c} \sim 10^{-10} \rho_{p l}$. This is not a problem neither for teleparallel LQC nor for our extrinsic curvature LQC because in both theories the critical density is only a parameter, however this is in contradiction with the value of the the critical energy density in holonomy corrected LQC which has the determined value $\rho_{c} \cong 0.4 \rho_{p l}$.

In the same way, the matter bounce scenario leads to a flat power spectrum, i.e., the spectral index of scalar perturbations is exactly equal to 1, which does not agree with the recent observational results provided by the joint analysis of BICEP2/Keck Array and Planck teams [101. This problem could easily overpassed assuming that the universe is not exactly matter dominated, rather with an Equation of State $P=-\epsilon \rho$ with $\epsilon \approx 0.003$ 98. However, the problem is not solved at all because, as we have already shown, in order to have a tensor/scalar ratio satisfying the constraint $r \leq 0.12$ we have to fine tune the value of the scalar field at the bounce. For this reason, a more sophisticated improvement seems necessary. One way comes from the so-called matter-ekpyrotic bounce scenario introduced in [104, developed in [105, 106], and studied for the particular of the LQC background in [100, 107. In this new scenario, the universe evolves at very early times in the contracting phase from a matter domination regime to an ekpyrotic one, which leads, in the case of the LQC background with the model proposed in [107, to a power spectrum of scalar perturbations of the order $H_{E}^{2} / M_{p l}^{2}$, where $H_{E}$ is the value of the Hubble parameter at the phase transition from matter domination to ekpyrotic phase. Moreover, in the same framework of LQC, the improved model studied in [100] leads to the same power spectrum of scalar perturbations as in [107, with a spectral index and running entering in its observational 1-dimensional marginalized domain at $2 \sigma$ C.L..

On the other hand, apart from solving the well-known Belinsky-Khalatnikov-Lifshitz (BKL) instability: the efective energy density of primordial anisotropy scales as $a^{-6}$ in the contracting phase [108], the more important thing is that the phase transition provides the mechanism to create enough particles, via gravitational particle production, to 
reheat and thermalize the universe in order to math with the hot Friedmann universe [109, 110].

\section{CONCLUSIONS}

We have presented a theory based in the ADM formalism where, given a pre-determined slicing, the Lagrangian of the gravitation sector is proportional to the intrinsic curvature plus a function $f$ depending on the extrinsic one. The a priori slicing is the price one has to pay in order to generalize, using the ADM formalism, GR to an $f$-theory, and similarly in teleparallelism where a fixed orthonormal basis in the tangent bundle has to be chosen, or in holonomy corrected LQC where the Asthekar connection is replaced by a convenient sinus function.

In the work we have tried to justify the choice of a preferred foliation based on an improvement of Weyl's postulate, where the 4-velocity of the preferred observers is the time-like eigenvector of the stress tensor, which goes precisely in the spirit of Weyl's postulate, where the matter content of the universe must fix a preferred frame. Fortunately, we have shown that the equations dealing with perturbations are gauge invariant, as in General Relativity, teleparallelism or holohomy corrected LQC.

At the level of the background, i.e., working in the flat FLRW spacetime with the usual co-moving frame, we show that in order to have a bounce, the use of multivalued functions $f$ is mandatory, and the simplest one is the one that leads to the holonomy corrected Friedmann equation in LQC. For a general metric, using the variational principle we have obtained, in the case of a general $f$-theory, the Hamiltonian and diffeomorphism constraints and the dynamical equations, which have been used to calculate the equations of perturbations in this theory.

We have compared them with the ones obtained in teleparallel $f(\mathcal{T})$ gravity, being $\mathcal{T}$ the torsion provided by the Weitzenbök connection, showing that for tensor perturbations the M-S equation coincides, and for scalar perturbations it only differs in the square of the velocity of sound. Moreover, for the particular case of the $f$ which leads to the same background of LQC, we have compared them with the perturbed equations of the holonomy corrected LQC, obtaining that for scalar perturbations, the M-S equation is the same, but for tensor perturbations it is completely different.

Finally, we have applied the above results to the matter bounce scenario in LQC, calculating the power spectrum for scalar and tensor perturbations, and showing numerically that, by choosing carefully some backgrounds, the ratio of tensor to scalar perturbations, namely $r$, satisfies the observational constraint $r \leq 0.12$ provided by the joint analysis of BICEP2/Keck Array and Planck teams [101.

\section{Acknowledgments}

This investigation has been supported in part by MINECO (Spain), projects MTM2017-84214-C2-1-P and MTM2015-69135-P, and by the Catalan Government projects 2017-SGR-247 and 2014-SGR-634. Finally, the authors are very grateful the reviewer for his constructive suggestions that result in an improved version of the manuscript both in quality as well as in presentation.

[1] M. Novello and S.E.P. Bergliaffa, Phys. Rept. 463, 127 (2008) arXiv:0802.1634

[2] R. Brandenberger, The Matter Bounce Alternative to Inflationary Cosmology, (2012) [arXiv: 1206.4196].

[3] R.H. Brandenberger, Int. J. Mod. Phys. Conf. Ser. 01, 67 (2011) arXiv:0902.4731.

[4] R.H. Brandenberger, AIP Conf. Proc. 1268, 3 (2010) arXiv:1003.1745.

[5] Y.F., Cai, Sci. China Phys. Mech. Astron. 57, 1414 (2014). arXiv:1405.1369 [hep-th]]

[6] A. Guth, Phys. Rev. D 23, 347 (1981).

[7] A. Linde, Phys. Lett. B 108, 389 (1982).

[8] A. Albrecht and P.J. Steinhardt, Phys. Rev. Lett. 48, 1220 (1982).

[9] M. Bojowald, G. Calcagni and S. Tsujikawa, JCAP 11, 046 (2011) arXiv:1107.1540.

[10] M. Bojowald, Comptes Rendus - Physique 16, 1012 (2015) arXiv:1509.01833.

[11] T. Zhu, A. Wang, G. Cleaver, K. Kirsten and Q. Sheng, Phys. Rev. D 96, 083520 (2017) arXiv:1705.07544.

[12] T. Zhu, A. Wang, K. Kirsten, G. Cleaver and Q. Sheng, Phys. Lett. B773, 196 (2017) arXiv:1607.06329.

[13] L.E. Allen and D. Wands, Phys. Rev. D 70, 063515 (2004) [arXiv:0404441]. 
[14] Y.-F. Cai, T. Qiu, Y.-S. Piao, M. Li and X. Zhang, JHEP 10, 071 (2007) arXiv:0704.1090.

[15] [8] T. Qiu, J. Evslin, Y.-F. Cai, M. Li and X. Zhang, JCAP 10, 036 (2011) arXiv:1108.0593.

[16] C. Lin, R.H. Brandenberger and L. Perreault Levasseur, JCAP 04, 019 (2011) arXiv:1007.2654.

[17] M. Roshan and F. Shojai, Phys. Rev. D 94, 044002 (2016) arXiv:1607.06049.

[18] K. Bamba, A. N. Makarenko, A. N. Myagky, S. Nojiri and S. D. Odintsov, JCAP 01, 008 (2014) arXiv:1309.3748].

[19] S. Nojiri, S.D. Odintsov and V.K. Oikonomou, Phys. Rev. D 93, 084050 (2016) arXiv:1601.04112.

[20] S.D. Odintsov and V.K. Oikonomou, Phys. Rev. D 91, 064036 (2015) arXiv:1502.06125.

[21] K. Bamba, A. N. Makarenko, A. N. Myagky and S. D. Odintsov, Physics Letters B 732, 349 (2014) arXiv:1403.3242.

[22] K. Bamba, A. N. Makarenko, A. N. Myagky and S. D. Odintsov, JCAP 04, 001 (2015) arXiv:1411.3852.

[23] S. Nojiri, S.D. Odintsov and V.K. Oikonomou, Phys. Rep. 692, 1 (2017) arXiv:1705.11098.

[24] J. Beltran Jimenez, L. Heisenberg, G. J. Olmo, and D. Rubiera-Garcia, Born-Infeld inspired modifications of gravity, (2017) arXiv:1704.03351.

[25] J. Beltran Jimenez, L. Heisenberg, G. J. Olmo, and D. Rubiera-Garcia, On gravitational waves in Born-Infeld inspired non-singular cosmologies, (2017) arXiv:1707.08953.

[26] A. Ashtekar, T. Pawlowski and P. Singh, Phys. Rev. D73, 124038 (2006) [arXiv:0604013]

[27] P. Singh, Phys. Rev. D73, 063508 (2006) [arXiv:0603043].

[28] P. Singh, K. Vandersloot and G. Vereshchagin, Phys. Rev. D74, 043510 (2006) [arXiv:0606032].

[29] P. Singh, J. Phys. Conf. Ser. 140, 012005 (2008) arXiv:0901.1301.

[30] F. Cianfrani and G. Montani, Phys. Rev. D82, 021501 (2010) arXiv:1006.1814].

[31] P. Dzierzak, P. Malkiewicz and W. Piechocki, Phys. Rev. D80, 104001 (2009) arXiv:0907.3436].

[32] P. Singh, Class. Quant. Grav. 26, 125005 (2009) arXiv:0901.2750.

[33] M. Sami, P. Singh and S. Tsujikawa, Phys. Rev. D74, 043514 (2006) [arXiv:0605113].

[34] T. Naskar and J. Ward, Phys. Rev. D76, 063514 (2007) arXiv:0704.3606.

[35] A. Corichi and P. Singh, Phys. Rev. D80, 044024 (2009) arXiv:0905.4949.

[36] G. Immirzi, Nucl. Phys. Proc. Suppl. 57, 65 (1997) [arXiv:9701052].

[37] A. Ashtekar and P. Singh, Class. Quantum Grav. 28, 213001 (2011) arXiv:1108.0893.

[38] K. Bamba, J. de Haro and S.D. Odintsov, JCAP 02, 008 (2013) arXiv:1211.2968.

[39] A. Ashtekar, T. Pawlowski and P. Singh, Phys. Rev. D74, 084003 (2006) [arXiv:0607039].

[40] P. Dzierzak, P. Malkiewicz and W. Piechocki, Phys. Rev. D80, 104001 (2009) arXiv:0907.3436 [gr-qc]].

[41] J. Haro and E. Elizalde, EPL 89, 69001 (2010).

[42] J. Amoros, J. de Haro and S.D. Odintsov, Phys. Rev. D87, 104037 (2013) arXiv:1305.2344.

[43] R.R. Caldwell, M. Kamionkowski and N.N. Weinberg, Phys. Rev. Lett. 91, 071301 (2003) [arXiv:0302506].

[44] P. H. Frampton, K. J. Ludwick and R. J. Scherrer, Phys.Rev. D84, 063003 (2011) arXiv:1106.4996.

[45] M. Bojowald and G.M. Hossain, Phys. Rev. D77, 023508 (2008) [arXiv: 0709.2365].

[46] E. Wilson-Ewing, Class. Quant. Grav. 29, 085005 (2012) arXiv:1108.6265.

[47] T. Cailleteau, J. Mielczarek, A. Barrau and J. Grain Class. Quantum Grav. 29, 095010 (2012) [arXiv: 1111.3535].

[48] J. Grain, A. Barrau and A. Gorecki Phys. Rev. D79 , 084015 (2009) [arXiv: 0902.3605].

[49] T. Cailleteau, A. Barrau, F. Vidotto and J. Grain, Phys. Rev. D86, 087301 (2012) [arXiv: 1206.6736].

[50] T. Cailleteau, L. Linsefors and A. Barrau, Class. Quant. Grav. 31, 125011 (2014) [arXiv: 1307.5238].

[51] R. C. Helling Higher curvature counter terms cause the bounce in loop cosmology, (2009) arXiv:0912.3011.

[52] G. Date and S. Sengupta, Class. Quant. Grav. 26 (2009) 105002, arXiv:0811.4023.

[53] J. de Haro, JCAP 1207, 007 (2012) arXiv:1204.5604.

[54] R. Weitzenböck, Invarianten Theorie, Noordhoff, Groningen, (1923).

[55] F.W. Hehl, P. Von Der Heyde, G. D. Kerlick, and J. M. Nester, Rev. Mod. Phys. 48, 393 (1976).

[56] R. Ferraro and F. Fiorini, Phys. Rev. D75, 084031 (2007) [arXiv:0610067].

[57] G. R. Bengochea and R. Ferraro, Phys. Rev. D79, 124019 (2009) arXiv:0812.1205.

[58] J. de Haro and J. Amoros, Phys. Rev. Lett. 110 , 071104 (2013) arXiv:1211.5336.

[59] Y-F. Cai, S-H. Chen, J.D. Dent, S. Dutta and E. N. Saridakis Class. Quantum Grav. 28, 215011 (2011) arXiv:1104.4349.

[60] S-H. Chen, J.D. Dent, S. Dutta and E. N. Saridakis, Phys. Rev. D83, 023508 (2011) [arXiv: 1008.1250].

[61] J. Haro, JCAP 1311, 068 (2013) [Erratum-ibid. 1405, E01 (2014)] [arXiv:1309.0352.

[62] J. de Haro and J. Amorós, JCAP 1408, 025 (2014) arXiv:1403.6396].

[63] J. Haro and J. Amorós, JCAP 1412, 031 (2014) arXiv:1406.0369.

[64] B. Li, T.P. Sotiriou and and J.D. Barrow, Phys. Rev. D83, 064035 (2011) arXiv:1010.1041.

[65] T.P. Sotiriou, B. Li and and J.D. Barrow, Phys. Rev. D83 , 104030 (2011) arXiv:1012.4039.

[66] R.L. Arnowitt, S. Deser and C.W.Misner, "The Dynamics of General Relativity" in Gravitation:an introduction to current research, Louis Witten ed., Wilew (1962), chap. 7, pp 227, [arXiv:0405109].

[67] E. Gourgoulhon, $3+1$ Formalism and Bases of Numerical Relativity, [arXiv:0703035].

[68] L. Smarr and J.W. York, Phys. Rev. D17, 2529 (1978).

[69] J.M. Bardeen, Gauge and radiation conditions in numerical relativity, in Rayonnement gravitationnel / Gravitation Radiation, edited by N. Deruelle and T. Piran, North Holland, Amsterdam p.433 (1983).

[70] J. E. Marsden and F. J. Tipler , Physics Reports 66, 109 (1980).

[71] N. O. Murchadha, Phys. Lett. A 77, 103 (1980).

[72] T. de Donder, Ann. Inst. H. Poincaré 1, 77 (1930).

[73] G.F.R. Ellis, R. Maartens and M.A.H. MacCallum, Relativistic Cosmology, Cambridge University Press (2012). 
[74] S. E. Rugh and H. Zinkernagel, Weyl's principle, cosmic time and quantum fundamentalism, (2010) arXiv:1006.5848].

[75] A. Riotto, Inflation and the Theory of Cosmological Perturbations, (2002) [arXiv:0210162].

[76] S.H. Hawking and G.F.R. Ellis, The large scale structure of space-time, Cambridge universe press (1973).

[77] R. Geroch, Domain of dependence, J. Math. Phys. 11, 437 (1970).

[78] S. E. Rugh and H. Zinkernagel, Stud. Hist. Philos. Mod. Phys. 40, 1 (2009) arXiv:0805.1947.

[79] G.F.R. Ellis, Space time and the passage of time, (2012) arXiv:1208.2611.

[80] S. E. Rugh and H. Zinkernagel, Limits of time in cosmology (2016) arXiv:1603.05449].

[81] S. Nojiri and S. D. Odintsov, Phys. Rept. 505, 59 (2011) arXiv:1011.0544.

[82] S. Nojiri and S. D. Odintsov, Int. J. Geom. Meth. Mod. Phys. 4, 115 (2007) [arXiv: 0601213].

[83] V.F. Mukhanov, Physical Foundations of Cosmology, Cambridge university press (2005).

[84] M. Ryan Jr., Hamiltonian Cosmology, Springer Verlag (1972).

[85] E. Pazos, Aplicación del formalismo Lagrangiano ADM a un modelo cosmológico Univ. de San Carlos de Guatemala, BS thesis (in Spanish) (2000).

[86] S. Chinaglia, A. Colleaux and S. Zerbini, Galaxies 5, 51 (2017) arXiv:1708.08667.

[87] T. Harko, F. S. N. Lobo, S. Nojiri and S. D. Odintsov, Phys. Rev. D84, 024020 (2011) arXiv:1104.2669.

[88] H. Shabani, and A. H. Ziaie, Bouncing cosmological solutions from $f(R, T)$ gravity (2017) arXiv:1708.07874.

[89] H. Velten, and T. R. P. Carames, Phys. Rev. D 95, 123536 (2017) arXiv:1702.07710.

[90] A. H. Chamseddine and V. Mukhanov, JCAP 1703, 009 (2017) arXiv:1612.05860.

[91] D. Langlois, H. Liu, K. Noui and E. Wilson-Ewing, Class. Quant. Grav. 34, 225004 (2017) arXiv:1703.10812.

[92] N. Bodendorfer, A. Schäfer and J. Schliemann, On the canonical structure of general relativity with a limiting curvature and its relation to loop quantum gravity, (2017) arXiv:1703.10670.

[93] J. de Haro and S. Pan, A note on bouncing backgrounds, (2018) arXiv:1801.05475].

[94] J.Z. Tang and Q. Xu, The Cosmological Constant as a Function of Extrinsic Curvature and Spatial Curvature (2010) arXiv:1008.0187.

[95] L. Lehner, Class. Quant. Grav. 18, R25, (2001) [arXiv:0106072].

[96] J. Isenberg, Initial Value Problem in General Relativity, (2013) arXiv:1304.1960.

[97] F. Mukhanov, H.A. Feldman and R.H. Brandenberger, Phys. Rep. 215, 203 (1992).

[98] E. Wilson-Ewing, JCAP 03, 026 (2013) arXiv:1211.6269].

[99] J. Mielczarek, Phys. Lett. B 675, 273 (2009) arXiv:0809.2469.

[100] J. Haro, J. Amorós and L. Aresté Saló, JCAP 09, 002 (2017) arXiv:1703.03710.

[101] P.A.R. Ade et al., Phys. Rev. Lett. 114, 101301 (2015) arXiv:1502.00612.

[102] WMAP collaboration, E. Komatsu et al., Astrophys. J. Suppl. 192, 18 (2011) arXiv:1001.4538 .

[103] K.A. Meissner, Class. Quant. Grav. 21, 5245 (2004) [arXiv:0407052].

[104] Y.-F. Cai, D.A. Easson and R. Brandenberger, JCAP 08, 020 (2012) arXiv:1206.2382.

[105] X. Gao, D. Langlois and S. Mizuno, JCAP 10, 023 (2013) arXiv:1306.5680.

[106] Y.-F. Cai, R. Brandenberger and P. Peter, Class. Quant. Grav. 30, 075019 (2013) arXiv:1301.4703.

[107] Y.-F. Cai and E. Wilson-Ewing, JCAP 03, 026 (2014) arXiv:1402.3009.

[108] V.A. Belinsky, I.M. Khalatnikov and E.M. Lifshitz, Adv. Phys. 19, 525 (1970).

[109] J. Quintin, Y.-F. Cai and R.H. Brandenberger, Phys. Rev. D 90, 063507 (2014) arXiv:1406.6049.

[110] J. Haro and E. Elizalde, JCAP 10, 028 (2015) arXiv:1505.07948. 